PROCEEDINGS OF THE

AMERICAN MATHEMATICAL SOCIETY

Volume 138, Number 4, April 2010, Pages 1349-1359

S 0002-9939(09)10220-4

Article electronically published on November 25, 2009

\title{
OPERATOR BIPROJECTIVITY OF COMPACT QUANTUM GROUPS
}

\author{
MATTHEW DAWS \\ (Communicated by Marius Junge)
}

\begin{abstract}
Given a (reduced) locally compact quantum group $A$, we can consider the convolution algebra $L^{1}(A)$ (which can be identified as the predual of the von Neumann algebra form of $A$ ). It is conjectured that $L^{1}(A)$ is operator biprojective if and only if $A$ is compact. The "only if" part always holds, and the "if" part holds for Kac algebras. We show that if the splitting morphism associated with $L^{1}(A)$ being biprojective can be chosen to be completely positive, or just contractive, then we already have a Kac algebra. We give another proof of the converse, indicating how modular properties of the Haar state seem to be important.
\end{abstract}

\section{INTRODUCTION}

A Banach algebra $A$ is biprojective if the multiplication map $\Delta_{*}: A \widehat{\otimes} A \rightarrow A$ has a right inverse in the category of $A$-bimodule maps. This can be thought of as a "finiteness condition". In particular, the group algebra $L^{1}(G)$ is biprojective if and only if $G$ is compact; see [9, Chapter IV, Theorem 5.13].

When dealing with more non-commutative (or "quantum") algebras (here we focus on $L^{1}(G)^{*}=L^{\infty}(G)$ when we suggest that the classical situation is commutative), there is a large amount of evidence that operator spaces form the correct category to work in. For example, if we consider the Fourier algebra $A(G)$, then $A(G)$ is operator biprojective if and only if $G$ is discrete, 23. When $G$ is abelian, as $A(G) \cong L^{1}(\hat{G})$, and $\hat{G}$ is compact if and only if $G$ is discrete, this result is in full agreement with what we might expect. By contrast, if we ask when $A(G)$ is biprojective, then, if $G$ is discrete and almost abelian (contains a finite-index abelian subgroup), then $A(G)$ is biprojective. Conversely, if $A(G)$ is biprojective, then $G$ is discrete, and either almost abelian, or is non-amenable yet does not contain $\mathbb{F}_{2}$; see [16].

In this paper, we shall continue the study of when the convolution algebra of a (reduced) compact quantum group is operator biprojective. It was shown in 1, Theorem 4.12] that if the convolution algebra of a locally compact quantum group $\mathbb{G}$ is operator biprojective, then $\mathbb{G}$ is already compact. Conversely, if $\mathbb{G}$ is a compact Kac algebra, then $\mathbb{G}$ is operator biprojective. We shall show that if the right inverse

Received by the editors May 16, 2009, and, in revised form, July 12, 2009.

2010 Mathematics Subject Classification. Primary 46L89, 46M10; Secondary 22D25, 46L07, 46L65, 47L25, 47L50, 81R15.

Key words and phrases. Compact quantum group, biprojective, Kac algebra, modular automorphism group.

(C)2009 American Mathematical Society Reverts to public domain 28 years from publication 1349 
to $\Delta_{*}$ can be chosen to be completely contractive, then $\mathbb{G}$ must already be a Kac algebra. We make some remarks on the general case. We indicate that the modular theory of the Haar state seems to be important outside of the Kac case, and it seems likely that a better understanding of how to deal with how the coproduct interacts with the modular automorphism group will be necessary to completely characterise when the convolution algebra of $\mathbb{G}$ is operator biprojective.

We shall follow the notation of $[\underline{6}$, and in particular, write $\widehat{\otimes}$ for the operator space projective tensor product, and write $\mathcal{C B}(E, F)$ to denote the space of complete bounded linear maps between the operator spaces $E$ and $F$.

We would like to thank the referee for helpful comments which have improved the exposition and for bringing [4] to our attention.

\section{LOCALLY COMPACT QUANTUM GROUPS}

Locally compact quantum groups [11, 10] are an axiomatic framework which encompasses the $L^{1}(G)$ algebras, the Fourier algebra $A(G)$, and various "quantum" examples, for example, Woronowicz's compact quantum groups. Kac algebras [7] are an earlier axiomatic framework which fails to encompass many of the "quantum" examples, for example [26].

However, we shall concentrate on the compact case, which is technically easier. We shall follow the presentation of [21, which in turn closely follows Woronowicz's original papers 24] and 25. See also readable, non-technical accounts in [10, and the survey [13, although be aware that these sources use different notation.

A compact quantum semigroup is a unital $C^{*}$-algebra $A$ equipped with a unital *-homomorphism $\Delta: A \rightarrow A \otimes_{\min } A$ such that $(\Delta \otimes \iota) \Delta=(\iota \otimes \Delta) \Delta$. A compact quantum group is a compact quantum semigroup $(A, \Delta)$ which satisfies the cancellation laws, namely that

$$
\Delta(A)(A \otimes 1):=\operatorname{lin}\{\Delta(a)(b \otimes 1): a, b \in A\}, \quad \Delta(A)(1 \otimes A)
$$

are both dense in $A \otimes_{\min } A$. If $G$ is a compact semigroup, then we may set $A=C(G)$ and $\Delta(f)(s, t)=f(s t)$ to get a compact quantum semigroup $(A, \Delta)$. Then the cancellation laws correspond to $G$ having the cancellation laws: namely that if $s t=s r$ for $s, t, r \in G$, then $t=r$, and similarly with the orders reversed. As sketched in [13, these are equivalent to $G$ being a group.

From now on, fix a compact quantum group $(A, \Delta)$. These axioms imply that $A$ carries a unique Haar state, that is, a state $\varphi \in A^{*}$ such that

$$
(\varphi \otimes \iota) \Delta(a)=\varphi(a) 1=(\iota \otimes \varphi) \Delta(a) \quad(a \in A) .
$$

We can form the GNS construction $(H, \Lambda)$ for $\varphi$. We shall always suppose that $(A, \Delta)$ is reduced, that is, that $\varphi$ is faithful. As such, we shall identify $A$ with a concrete $C^{*}$-algebra acting on $H$. If $\varphi$ is not faithful, then we may quotient by its kernel $N=\left\{a \in A: \varphi\left(a^{*} a\right)=0\right\}$ to obtain a reduced compact quantum group. Note that $N$ is an ideal because $\varphi$ is a KMS weight (see below); see the details in [3. Theorem 2.1].

Let $M=A^{\prime \prime}$ be the von Neumann algebra generated by $A$. Then $\Delta$ extends to a normal $*$-homomorphism $\Delta: M \rightarrow M \bar{\otimes} M$. Then, by [6, Theorem 7.2.4], $(M \bar{\otimes} M)_{*}=M_{*} \widehat{\otimes} M_{*}$ and normality of $\Delta$ induces a complete contraction $\Delta_{*}: M_{*} \widehat{\otimes} M_{*} \rightarrow M_{*}$. That $\Delta$ is coassociative implies that $\Delta_{*}$ is associative, so $M_{*}$ becomes a completely contractive Banach algebra. If we started with a compact 
group $G$, then $M_{*}$ is nothing but $L^{1}(G)$, and so we refer to $M_{*}$ as the convolution algebra of $(A, \Delta)$. For more on (locally) compact quantum groups in the von Neumann algebra setting, see [12].

A finite-dimensional corepresentation of $(A, \Delta)$ is an invertible matrix $u=$ $\left(u_{i, j}\right) \in \mathbb{M}_{n}(A)$ such that

$$
\Delta\left(u_{i j}\right)=\sum_{k=1}^{n} u_{i k} \otimes u_{k j} \quad(1 \leq i, j \leq n) .
$$

There are suitable notions of intertwiner between corepresentations, and what an irreducible corepresentation is. Every finite-dimensional corepresentation can be written as the direct sum of irreducible corepresentations. Using the Haar state, it can be shown that every finite-dimensional corepresentation is equivalent to a unitary one, that is, where $u \in \mathbb{M}_{n}(A)$ is unitary. The general corepresentation theory of $(A, \Delta)$ parallels the representation theory of compact groups very closely.

Let $\left\{u^{\alpha}=\left(u_{i j}^{\alpha}\right)_{i, j=1}^{n_{\alpha}}: \alpha \in \mathbb{A}\right\}$ be a maximal family of mutually inequivalent, finite-dimensional irreducible unitary corepresentations of $(A, \Delta)$. Let $\alpha_{0} \in \mathbb{A}$ be such that $v^{\alpha_{0}}=1$, the trivial corepresentation. Let $\mathcal{A}$ be the algebra generated by $\left\{u_{i j}^{\alpha}: \alpha \in \mathbb{A}, 1 \leq i, j \leq n_{\alpha}\right\}$ in $A$. Then $\mathcal{A}$ is a Hopf $*$-algebra, and $\left\{u_{i j}^{\alpha}: \alpha \in\right.$ $\left.\mathbb{A}, 1 \leq i, j \leq n_{\alpha}\right\}$ forms a basis for $\mathcal{A}$. This means that $\mathcal{A}$ is a $*$-algebra, that $\Delta$ restricts to give a $*$-homomorphism $\Delta: \mathcal{A} \rightarrow \mathcal{A} \otimes \mathcal{A}$ (the algebraic tensor product) and there exist maps $\epsilon: \mathcal{A} \rightarrow \mathbb{C}$ and $S: \mathcal{A} \rightarrow \mathcal{A}$, the counit and antipode, satisfying the usual properties. Indeed, for $\alpha \in \mathbb{A}$ and $1 \leq i, j \leq n_{\alpha}$, we have that

$$
\Delta\left(u_{i, j}^{\alpha}\right)=\sum_{k=1}^{n_{\alpha}} u_{i, k}^{\alpha} \otimes u_{k, j}^{\alpha}, \quad S\left(u_{i, j}^{\alpha}\right)=\left(u_{j, i}^{\alpha}\right)^{*}, \quad \epsilon\left(u_{i, j}^{\alpha}\right)=\delta_{i j}, \quad \varphi\left(u_{i, j}^{\alpha}\right)=\delta_{\alpha, \alpha_{0}} .
$$

Furthermore, for each $\alpha \in \mathbb{A}$, there exists a unique positive invertible matrix $F^{\alpha} \in$ $\mathbb{M}_{n_{\alpha}}$ with $\operatorname{Tr} F^{\alpha}=\operatorname{Tr}\left(F^{\alpha}\right)^{-1}$, and such that

$$
\varphi\left(\left(u_{i j}^{\beta}\right)^{*} u_{k l}^{\alpha}\right)=\delta_{\alpha \beta} \delta_{j l} \frac{\left(\left(F^{\alpha}\right)^{-1}\right)_{k i}}{\operatorname{Tr}\left(F^{\alpha}\right)}, \quad \varphi\left(u_{i j}^{\beta}\left(u_{k l}^{\alpha}\right)^{*}\right)=\delta_{\alpha \beta} \delta_{i k} \frac{F_{l j}^{\alpha}}{\operatorname{Tr}\left(F^{\alpha}\right)} .
$$

The Hopf *-algebra $\mathcal{A}$ is norm dense in $A$ and is the unique such dense Hopf *algebra; see [3, Appendix A].

These " $F$-matrices" allow us to define characters on $\mathcal{A}$. For $z \in \mathbb{C}$, define

$$
f_{z}: \mathcal{A} \rightarrow \mathbb{C}, \quad u_{i j}^{\alpha} \mapsto\left(\left(F^{\alpha}\right)^{z}\right)_{i j} .
$$

As $F^{\alpha}$ is positive, the matrix $\left(F^{\alpha}\right)^{z}$ makes sense. Then, for $w, z \in \mathbb{C}$, define

$$
\rho_{z, w}: \mathcal{A} \rightarrow \mathcal{A}, \quad u_{i j}^{\alpha} \mapsto \sum_{k, l=1}^{n_{\alpha}} f_{w}\left(u_{i k}^{\alpha}\right) f_{z}\left(u_{l j}^{\alpha}\right) u_{k l}^{\alpha} .
$$

Then $\rho_{z, w}$ is an automorphism of $\mathcal{A}$ with inverse $\rho_{-z,-w}$, and if $z$ and $w$ are purely imaginary, then $\rho_{z, w}$ is a $*$-automorphism of $\mathcal{A}$.

In particular, set

$$
\sigma_{z}=\rho_{i z, i z}, \quad \tau_{z}=\rho_{-i z, i z} \quad(z \in \mathbb{C}) .
$$

Then $\left(\sigma_{t}\right)_{t \in \mathbb{R}}$ is the (restriction) of the modular automorphism group for $\varphi$, and $\left(\tau_{t}\right)_{t \in \mathbb{R}}$ is the (restriction) of the scaling group. For example, we can calculate that $\varphi\left(a \sigma_{-i}(b)\right)=\varphi(b a)$ for $a, b \in \mathcal{A}$, a relation which we expect, as $\varphi$ is KMS for $\sigma$. 
See [20] for more details on the modular theory of weights on general von Neumann algebras, and [11, 24] and [25] for the specific theory as applied to compact quantum groups (and for details of the scaling group).

Proposition 2.1. There exists a maximal family of mutually inequivalent, finitedimensional irreducible unitary corepresentations of $(A, \Delta)$, say $\left\{v^{\alpha}=\left(v_{i j}^{\alpha}\right)_{i, j=1}^{n_{\alpha}}\right.$ : $\alpha \in \mathbb{A}\}$, with the property that the associated $F$-matrices are all diagonal, say $F^{\alpha}$ has diagonal entries $\left(\lambda_{i}^{\alpha}\right)_{i=1}^{n_{\alpha}}$, so that $\sum_{i} \lambda_{i}^{\alpha}=\sum_{i}\left(\lambda_{i}^{\alpha}\right)^{-1}=T r_{\alpha}$, say.

Proof. Start with some maximal family $\left\{u^{\alpha}=\left(u_{i j}^{\alpha}\right)_{i, j=1}^{n_{\alpha}}: \alpha \in \mathbb{A}\right\}$ as before. As each $F^{\alpha}$ is positive it can be diagonalised by some unitary matrix $Q^{\alpha} \in \mathbb{M}_{n_{\alpha}}$. Let $\left(\lambda_{i}^{\alpha}\right)_{i=1}^{n_{\alpha}}$ be the eigenvalues of $F^{\alpha}$, so that $\operatorname{Tr}\left(F^{\alpha}\right)=\sum_{i} \lambda_{i}^{\alpha}=\operatorname{Tr}\left(\left(F^{\alpha}\right)^{-1}\right)=\sum_{i}\left(\lambda_{i}^{\alpha}\right)^{-1}$. Then $\left(Q^{\alpha}\right)^{*} F^{\alpha} Q^{\alpha}$ is the diagonal matrix with entries $\left(\lambda_{i}^{\alpha}\right)_{i=1}^{n_{\alpha}}$. Set

$$
v_{i j}^{\alpha}=\left(\left(Q^{\alpha}\right)^{*} u^{\alpha} Q^{\alpha}\right)_{i j}=\sum_{k, l=1}^{n_{\alpha}} \overline{Q_{k i}^{\alpha}} u_{k l}^{\alpha} Q_{l j}^{\alpha} \quad\left(\alpha \in \mathbb{A}, 1 \leq i, j \leq n_{\alpha}\right) .
$$

It is now routine to check that $v^{\alpha}$ is a unitary corepresentation matrix, and that the properties above still hold for the family $\left\{v_{i j}^{\alpha}\right\}$. For example, we see that

$$
\begin{aligned}
\varphi\left(\left(v_{i j}^{\beta}\right)^{*} v_{k l}^{\alpha}\right) & =\varphi\left(\left(\sum_{r, s} \overline{Q_{r i}^{\beta}} u_{r s}^{\beta} Q_{s j}^{\beta}\right)^{*} \sum_{t, p} \overline{Q_{t k}^{\alpha}} u_{t p}^{\alpha} Q_{p l}^{\alpha}\right) \\
& =\sum_{r, s, t, p} Q_{r i}^{\beta} \overline{Q_{s j}^{\beta}} \overline{Q_{t k}^{\alpha}} Q_{p l}^{\alpha} \varphi\left(\left(u_{r s}^{\beta}\right)^{*} u_{t p}^{\alpha}\right) \\
& =\delta_{\alpha \beta} \frac{1}{\operatorname{Tr}\left(F^{\alpha}\right)} \sum_{r, s, t} Q_{r i}^{\beta} \overline{Q_{s j}^{\beta}} \overline{Q_{t k}^{\alpha}} Q_{s l}^{\alpha}\left(\left(F^{\alpha}\right)^{-1}\right)_{t r} \\
& =\delta_{\alpha \beta} \frac{1}{\operatorname{Tr}_{\alpha}} \sum_{s}\left(Q^{\alpha}\right)_{j s}^{*} Q_{s l}^{\alpha}\left(\left(Q^{\alpha}\right)^{*}\left(F^{\alpha}\right)^{-1} Q^{\alpha}\right)_{k i}=\delta_{\alpha \beta} \delta_{j l} \delta_{k i} \frac{1}{\operatorname{Tr}_{\alpha}} \frac{1}{\lambda_{i}^{\alpha}} .
\end{aligned}
$$

Similar calculations show that

$$
\varphi\left(v_{i j}^{\beta}\left(v_{k l}^{\alpha}\right)^{*}\right)=\delta_{\alpha \beta} \delta_{i k} \delta_{j l} \frac{\lambda_{j}^{\alpha}}{\operatorname{Tr}_{\alpha}}
$$

and also

$$
f_{z}\left(v_{i j}^{\alpha}\right)=\delta_{i j}\left(\lambda_{i}^{\alpha}\right)^{z}, \quad \rho_{z, w}\left(v_{i j}^{\alpha}\right)=\left(\lambda_{i}^{\alpha}\right)^{w}\left(\lambda_{j}^{\alpha}\right)^{z} v_{i j}^{\alpha} .
$$

Henceforth, the family of corepresentations shall always be given by this proposition; notice in particular that $\left\{v_{i j}^{\alpha}: \alpha \in \mathbb{A}, 1 \leq i, j \leq n_{\alpha}\right\}$ forms a linear basis for $\mathcal{A}$.

\section{BipRojeCtivity}

Let $(A, \Delta)$ be a reduced compact quantum group, with associated Haar state $\varphi$, GNS construction $(H, \Lambda)$, von Neumann algebra $M$ and convolution algebra $M_{*}$. We shall study when $M_{*}$ is operator biprojective, that is, whether there is a completely bounded right inverse to $\Delta_{*}: M_{*} \widehat{\otimes} M_{*} \rightarrow M_{*}$ which is also an $M_{*^{-}}$ bimodule homomorphism. Henceforth, we shall term such a map $\theta_{*}$ a splitting morphism.

See [1, 2] for further details on the operator space case, and see [9, Chapter IV] or [17, Section 4.3] for the classical Banach space setting. 
Lemma 3.1. $M_{*}$ is biprojective if and only if there exists a normal completely bounded map $\theta: M \bar{\otimes} M \rightarrow M$ with

$$
\theta \Delta=\mathrm{id}, \quad \Delta \theta=(\theta \otimes \mathrm{id})(\mathrm{id} \otimes \Delta)=(\mathrm{id} \otimes \theta)(\Delta \otimes \mathrm{id}) .
$$

Proof. Suppose that such a $\theta$ exists; then as $\theta$ is normal, there exists $\theta_{*}: M_{*} \rightarrow$ $M_{*} \widehat{\otimes} M_{*}$ with $\Delta_{*} \theta_{*}=$ id. Then, for $\omega, \tau \in M_{*}$ and $x \in M$,

$$
\begin{aligned}
\left\langle x, \theta_{*}(\omega * \tau)\right\rangle & =\left\langle\theta(x), \Delta_{*}(\omega \otimes \tau)\right\rangle=\langle(\theta \otimes \mathrm{id})(\mathrm{id} \otimes \Delta)(x), \omega \otimes \tau\rangle \\
& =\left\langle(\operatorname{id} \otimes \Delta)(x), \theta_{*}(\omega) \otimes \tau\right\rangle=\left\langle x, \theta_{*}(\omega) * \tau\right\rangle .
\end{aligned}
$$

Here we write $*$ for both the product in $M_{*}$, and the bimodule action of $M_{*}$ on $M_{*} \widehat{\otimes} M_{*}$. Similarly, $\theta_{*}(\omega * \tau)=\omega * \theta_{*}(\tau)$, so we see that $\theta_{*}$ is an $M_{*}$-bimodule homomorphism.

The converse is simply a case of reversing the argument.

In the following section, we shall carefully study the structure of normal completely bounded maps $M \bar{\otimes} M \rightarrow M$. From now on, fix such a map $\theta: M \bar{\otimes} M \rightarrow M$ and let $\left\{\left(v_{i j}^{\alpha}\right)_{i, j=1}^{n_{\alpha}}: \alpha \in \mathbb{A}\right\}$ be as in Proposition 2.1.

Proposition 3.2. We have that $\theta \Delta=$ id and $\Delta \theta=(\theta \otimes \mathrm{id})(\mathrm{id} \otimes \Delta)=(\operatorname{id} \otimes \theta)(\Delta \otimes$ id) if and only if there exists a family $\left\{X^{\alpha} \in \mathbb{M}_{n_{\alpha}}: \alpha \in \mathbb{A}\right\}$ such that, for $\alpha, \beta \in \mathbb{A}$, $1 \leq i, j \leq n_{\alpha}$ and $1 \leq k, l \leq n_{\beta}$,

$$
\theta\left(v_{i j}^{\alpha} \otimes v_{k l}^{\beta}\right)=\delta_{\alpha \beta} X_{j k}^{\alpha} v_{i l}^{\alpha}, \quad \sum_{r=1}^{n_{\alpha}} X_{r r}^{\alpha}=1 .
$$

Proof. The "if" part follows as $\mathcal{A}$ generates $M$ and $\theta$ is normal.

Conversely, let $x \in M$ and $\alpha \in \mathbb{A}$. For $1 \leq i, j \leq n_{\alpha}$,

$$
\Delta \theta\left(x \otimes v_{i j}^{\alpha}\right)=(\theta \otimes \mathrm{id})(\mathrm{id} \otimes \Delta)\left(x \otimes v_{i j}^{\alpha}\right)=\sum_{r=1}^{n_{\alpha}} \theta\left(x \otimes v_{i r}^{\alpha}\right) \otimes v_{r j}^{\alpha} .
$$

Let $a_{i j}=\theta\left(x \otimes v_{i j}^{\alpha}\right)$, so that $\Delta\left(a_{i j}\right)=\sum_{r} a_{i r} \otimes v_{r j}^{\alpha}$. As $\Delta$ is a $*$-homomorphism, for $1 \leq k, l \leq n_{\alpha}$, we have that

$$
\Delta\left(a_{i j}\left(v_{k l}^{\alpha}\right)^{*}\right)=\sum_{r, s=1}^{n_{\alpha}} a_{i r}\left(v_{k s}^{\alpha}\right)^{*} \otimes v_{r j}^{\alpha}\left(v_{s l}^{\alpha}\right)^{*} .
$$

Applying $(\iota \otimes \varphi)$, we see that, by the calculations in Proposition 2.1

$$
\varphi\left(a_{i j}\left(v_{k l}^{\alpha}\right)^{*}\right) 1=\sum_{r, s=1}^{n_{\alpha}} a_{i r}\left(v_{k s}^{\alpha}\right)^{*} \varphi\left(v_{r j}^{\alpha}\left(v_{s l}^{\alpha}\right)^{*}\right)=\delta_{j l} \frac{\lambda_{j}^{\alpha}}{\operatorname{Tr}_{\alpha}} \sum_{r=1}^{n_{\alpha}} a_{i r}\left(v_{k r}^{\alpha}\right)^{*} .
$$

As $v^{\alpha}$ is a unitary matrix, we see that $\sum_{k}\left(v_{k r}^{\alpha}\right)^{*} v_{k s}^{\alpha}=\delta_{r s} 1$ for $1 \leq r, s \leq n_{\alpha}$. Thus

$$
\sum_{k=1}^{n_{\alpha}} \varphi\left(a_{i j}\left(v_{k l}^{\alpha}\right)^{*}\right) v_{k s}^{\alpha}=\delta_{j l} \frac{\lambda_{j}^{\alpha}}{\operatorname{Tr}_{\alpha}} \sum_{r, k=1}^{n_{\alpha}} a_{i r}\left(v_{k r}^{\alpha}\right)^{*} v_{k s}^{\alpha}=\delta_{j l} \frac{\lambda_{j}^{\alpha}}{\operatorname{Tr}_{\alpha}} a_{i s} .
$$

It follows that

$$
a_{i s}=\frac{\operatorname{Tr}_{\alpha}}{\lambda_{j}^{\alpha}} \sum_{k=1}^{n_{\alpha}} \varphi\left(a_{i j}\left(v_{k j}^{\alpha}\right)^{*}\right) v_{k s}^{\alpha} \quad\left(\alpha \in \mathbb{A}, 1 \leq i, j, s \leq n_{\alpha}\right) .
$$


Similarly, if we set $b_{i j}=\theta\left(v_{i j}^{\alpha} \otimes x\right)$, then $\Delta\left(b_{i j}\right)=\sum_{r} v_{i r}^{\alpha} \otimes b_{r j}$, and we can show that

$$
b_{s j}=\lambda_{i}^{\alpha} \operatorname{Tr}_{\alpha} \sum_{k=1}^{n_{\alpha}} \varphi\left(\left(v_{i k}^{\alpha}\right)^{*} b_{i j}\right) v_{s k}^{\alpha} \quad\left(\alpha \in \mathbb{A}, 1 \leq i, j, s \leq n_{\alpha}\right) .
$$

In particular, we see that $\theta\left(v_{i j}^{\alpha} \otimes v_{k l}^{\beta}\right)$ is in the linear span of $\left\{v_{i s}^{\alpha}: 1 \leq s \leq n_{\alpha}\right\}$ and the linear span of $\left\{v_{r l}^{\beta}: 1 \leq r \leq n_{\beta}\right\}$. Hence $\theta\left(v_{i j}^{\alpha} \otimes v_{k l}^{\beta}\right)=0$ if $\alpha \neq \beta$. If $\alpha=\beta$, then by linear independence, we see immediately that

$$
\theta\left(v_{i j}^{\alpha} \otimes v_{k l}^{\alpha}\right)=X_{j k}^{\alpha} v_{i l}^{\alpha},
$$

for some scalar $X_{j k}^{\alpha}$. Finally, as $\sum_{k} \theta\left(v_{i k}^{\alpha} \otimes v_{k j}^{\alpha}\right)=v_{i j}^{\alpha}$, it follows that $\sum_{k} X_{k k}^{\alpha}=1$, as required.

Theorem 3.3. Let $(A, \Delta)$ be a compact quantum group with associated von Neumann algebra $M$. Let $\theta_{*}: M_{*} \rightarrow M_{*} \widehat{\otimes} M_{*}$ be a splitting morphism, and suppose further that $\theta=\theta_{*}^{*}$ is an $M$-bimodule map, in the sense that $\theta(\Delta(a) x \Delta(b))=a \theta(x) b$ for $x \in M \bar{\otimes} M$ and $a, b \in M$. Then the Haar state $\varphi$ is tracial, so $(M, \Delta)$ is a Kac algebra.

Proof. Let $\alpha \in \mathbb{A}$ and $1 \leq i, j, k \leq n_{\alpha}$. As $\theta(x \Delta(b))=\theta(x) b$ for $x \in M \bar{\otimes} M$ and $b \in M$, using the notation of the last proposition, we see that

$$
X_{j k}^{\alpha} v_{i j}^{\alpha}\left(v_{i j}^{\alpha}\right)^{*}=\theta\left(v_{i j}^{\alpha} \otimes v_{k j}^{\alpha}\right)\left(v_{i j}^{\alpha}\right)^{*}=\sum_{l=1}^{n_{\alpha}} \theta\left(v_{i j}^{\alpha}\left(v_{i l}^{\alpha}\right)^{*} \otimes v_{k j}^{\alpha}\left(v_{l j}^{\alpha}\right)^{*}\right) .
$$

Now, as $\left\{v_{r s}^{\beta}\right\}$ forms a basis for the $*$-algebra $\mathcal{A}$, and as $\varphi$ picks out the trivial corepresentation $v^{\alpha_{0}}=1$, by the calculations of Proposition 2.1 we see that

$$
v_{i j}^{\alpha}\left(v_{i l}^{\alpha}\right)^{*} \otimes v_{k j}^{\alpha}\left(v_{l j}^{\alpha}\right)^{*}=\delta_{j l} \frac{\lambda_{j}^{\alpha}}{\operatorname{Tr}_{\alpha}} 1 \otimes \delta_{k l} \frac{\lambda_{j}^{\alpha}}{\operatorname{Tr}_{\alpha}} 1+\text { other terms. }
$$

By "other terms", we are thinking of expanding using the basis $\left\{v_{r s}^{\beta}\right\}$ for $\mathcal{A}$; in particular, all these elements are in $\operatorname{ker} \varphi \otimes \operatorname{ker} \varphi$. The last proposition shows in particular that $\varphi \theta=\varphi \otimes \varphi$, and hence it follows that

$$
\sum_{l=1}^{n_{\alpha}} \varphi \theta\left(v_{i j}^{\alpha}\left(v_{i l}^{\alpha}\right)^{*} \otimes v_{k j}^{\alpha}\left(v_{l j}^{\alpha}\right)^{*}\right)=\delta_{j k}\left(\frac{\lambda_{j}^{\alpha}}{\operatorname{Tr}_{\alpha}}\right)^{2} .
$$

By applying $\varphi$ to (3.1), we conclude that

$$
X_{j k}^{\alpha} \frac{\lambda_{j}^{\alpha}}{\operatorname{Tr}_{\alpha}}=\delta_{j k}\left(\frac{\lambda_{j}^{\alpha}}{\operatorname{Tr}_{\alpha}}\right)^{2} \text { so that } X_{j k}^{\alpha}=\delta_{j k} \frac{\lambda_{j}^{\alpha}}{\operatorname{Tr}_{\alpha}} .
$$

We now repeat this argument on the right, so we find that

$$
\begin{aligned}
X_{j k}^{\alpha}\left(v_{i j}^{\alpha}\right)^{*} v_{i j}^{\alpha} & =\left(v_{i j}^{\alpha}\right)^{*} \theta\left(v_{i j}^{\alpha} \otimes v_{k j}^{\alpha}\right)=\sum_{s} \theta\left(\left(v_{i s}^{\alpha}\right)^{*} v_{i j}^{\alpha} \otimes\left(v_{s j}^{\alpha}\right)^{*} v_{k j}^{\alpha}\right) \\
& =\sum_{s} \delta_{s j} \frac{1}{\lambda_{i}^{\alpha} \operatorname{Tr}_{\alpha}} \delta_{s k} \frac{1}{\lambda_{k}^{\alpha} \operatorname{Tr}_{\alpha}} 1+\text { other terms. }
\end{aligned}
$$

Again, by applying $\varphi$ we see that

$$
X_{j k}^{\alpha} \frac{1}{\lambda_{i}^{\alpha} \operatorname{Tr}_{\alpha}}=\delta_{j k} \frac{1}{\lambda_{i}^{\alpha} \operatorname{Tr}_{\alpha}} \frac{1}{\lambda_{k}^{\alpha} \operatorname{Tr}_{\alpha}} \quad \text { so that } \quad X_{j k}^{\alpha}=\delta_{j k} \frac{1}{\lambda_{k}^{\alpha} \operatorname{Tr}_{\alpha}} .
$$


Hence we see that for all $\alpha$ and $1 \leq k \leq n_{\alpha}$, we have $\lambda_{k}^{\alpha}=1 / \lambda_{k}^{\alpha}$. As $\lambda_{k}^{\alpha}>0$, we see that $\lambda_{k}^{\alpha}=1$. In particular, the modular automorphism group $\sigma$ is trivial, and so $\varphi$ is tracial, as claimed.

Indeed, if $\varphi$ is tracial, then from Proposition 2.1 we see that $\lambda_{j}^{\alpha}=\left(\lambda_{i}^{\alpha}\right)^{-1}$ for all $i, j$. Thus $\lambda_{i}^{\alpha}=1$ for all $i$ and $\alpha$. It follows that the automorphisms $\rho_{z, w}$ are trivial, and hence also the scaling group is trivial. So the antipode $S$ is bounded; see also [24, Theorem 2.5]. It is now easy to verify the axioms of a compact Kac algebra; see [7, Section 6.2].

We note that an argument of Soltan ([18, Remark A.2]) shows that if a compact quantum group $(A, \Delta)$ has a faithful family of tracial states (that is, for non-zero $x \in A$ there is a tracial state $\phi$ with $\left.\phi\left(x^{*} x\right) \neq 0\right)$, then $(M, \Delta)$ is a Kac algebra.

Theorem 3.4. Let $(A, \Delta)$ be a compact quantum group with associated von Neumann algebra $M$. Let $\theta_{*}: M_{*} \rightarrow M_{*} \widehat{\otimes} M_{*}$ be a splitting morphism. Suppose that $\theta=\theta_{*}^{*}$ is completely positive or that $\Delta \theta$ is a contraction. Then $(M, \Delta)$ is a Kac algebra.

Proof. As $\theta(1)=\theta \Delta(1)=1$, if $\theta$ is positive, then $\theta$ is contractive, so $\Delta \theta$ is contractive.

We have that $\Delta \theta: M \bar{\otimes} M \rightarrow M \bar{\otimes} M$ is contractive and is a projection of $M \bar{\otimes} M$ onto the subalgebra $\Delta(M)$. A result of Tomiyama (22] or [19, Theorem 3.4, Chapter III]) tells us that, in particular, $\Delta \theta(\Delta(a) x \Delta(b))=\Delta(a) \theta(x) \Delta(b)$ for $a, b \in M$ and $x \in M \bar{\otimes} M$. As $\Delta$ is an injective homomorphism, the above theorem applies.

In the following section, we shall show the converse to this corollary: namely, that for a compact Kac algebra $(M, \Delta)$, we can choose $\theta$ to be a complete contraction; alternatively, see [15] or [1].

It is shown in [5] that if we have a completely bounded map $\theta: M \bar{\otimes} M \rightarrow M$ with $\theta \Delta=$ id, then there exists a completely bounded map $\theta_{1}: M \bar{\otimes} M \rightarrow M$ which is an $M$-bimodule map, in the above sense. However, there is no reason that $\theta_{1}$ need be normal, and no reason that the other conditions on $\theta$ will carry over to $\theta_{1}$, so that Proposition 3.2 need not apply to $\theta_{1}$. We can even choose $\theta_{1}$ to be completely positive, which were it also faithful would imply, by [20, Theorem 4.2, Chapter IX], the existence of a weight $\omega$ on $M \bar{\otimes} M$ with interesting modular properties. Again, there seems to be no reason to expect that we can choose $\theta_{1}$ in such a way.

\section{Completely Bounded maps}

There is a well-known structure theory for completely bounded maps [6. Section 5.3]. If $\theta: N \rightarrow M \subseteq \mathcal{B}(H)$ is a completely positive normal map between von Neumann algebras, then the usual proof of the Stinespring theorem (for example, [19, Chapter IV, Theorem 3.6]) can be adapted to show that there exists a Hilbert space $K$, a normal $*$-homomorphism $\pi: N \rightarrow \mathcal{B}(K)$ and a bounded map $U: H \rightarrow K$ such that $\theta(x)=U^{*} \pi(x) U$ for $x \in N$.

Showing the same for completely bounded maps is not quite as simple, but the details are worked out in, for example, the proof of [8, Theorem 2.4]. In particular, given $\theta: N \rightarrow M \subseteq \mathcal{B}(H)$, a completely contractive normal map between von Neumann algebras, there exist unital completely positive normal maps $\phi_{1}, \phi_{2}: N \rightarrow M$ 
such that

$$
\sigma: \mathbb{M}_{2}(N) \rightarrow \mathbb{M}_{2}(M) ; \quad\left(\begin{array}{ll}
a & b \\
c & d
\end{array}\right) \mapsto\left(\begin{array}{cc}
\phi_{1}(a) & \theta\left(b^{*}\right)^{*} \\
\theta(c) & \phi_{2}(d)
\end{array}\right)
$$

is unital completely positive and normal. One can now follow the presentation in 6. Theorem 5.33] or 14, essentially applying the Stinespring construction to $\sigma$. This yields a Hilbert space $K$, a normal *-homomorphism $\rho: \mathbb{M}_{2}(N) \rightarrow \mathcal{B}(K)$ and an isometry $U: H^{2} \rightarrow K$ such that $\sigma(x)=U^{*} \rho(x) U$ for $x \in \mathbb{M}_{2}(N)$. Following the proof of [8, Theorem 2.3], there also exists a normal $*$-homomorphism $\rho^{\prime}$ : $\mathbb{M}_{2}(M)^{\prime} \rightarrow \rho\left(\mathbb{M}_{2}(N)\right)^{\prime}$ such that $\rho^{\prime}(y) U=U y$ for $y \in \mathbb{M}_{2}(M)^{\prime}$.

Define $\pi: M \bar{\otimes} M \rightarrow \mathcal{B}(K), \pi^{\prime}: M^{\prime} \rightarrow \mathcal{B}(K)$, and $R, T: H \rightarrow K$ by

$\pi(x)=\rho\left(\begin{array}{ll}x & 0 \\ 0 & x\end{array}\right), \pi^{\prime}(y)=\rho^{\prime}\left(\begin{array}{ll}y & 0 \\ 0 & y\end{array}\right), T(\xi)=\rho\left(\begin{array}{ll}0 & 0 \\ 1 & 0\end{array}\right) U\left(\begin{array}{l}\xi \\ 0\end{array}\right), R(\xi)=U\left(\begin{array}{l}0 \\ \xi\end{array}\right)$,

for $x \in M \bar{\otimes} M, y \in M^{\prime}$ and $\xi \in H$. So $\pi$ and $\pi^{\prime}$ are normal $*$-homomorphisms, and $R$ and $T$ are contractions. Then, for $x \in M \bar{\otimes} M$ and $\xi, \eta \in H$,

$$
\begin{aligned}
\left(R^{*} \pi(x) T \xi \mid \eta\right) & =\left(\rho\left(\begin{array}{ll}
x & 0 \\
0 & x
\end{array}\right) \rho\left(\begin{array}{ll}
0 & 0 \\
1 & 0
\end{array}\right) U\left(\begin{array}{l}
\xi \\
0
\end{array}\right) \mid U\left(\begin{array}{l}
0 \\
\eta
\end{array}\right)\right) \\
& =\left(\sigma\left(\begin{array}{ll}
0 & 0 \\
x & 0
\end{array}\right)\left(\begin{array}{l}
\xi \\
0
\end{array}\right) \mid\left(\begin{array}{l}
0 \\
\eta
\end{array}\right)\right)=(\theta(x) \xi \mid \eta) .
\end{aligned}
$$

So $\theta(x)=R^{*} \pi(x) T$. Then also, for $y \in M^{\prime}$ and $\xi \in H$,

$$
T y \xi=\rho\left(\begin{array}{ll}
0 & 0 \\
1 & 0
\end{array}\right) U\left(\begin{array}{ll}
y & 0 \\
0 & y
\end{array}\right)\left(\begin{array}{l}
\xi \\
0
\end{array}\right)=\rho\left(\begin{array}{ll}
0 & 0 \\
1 & 0
\end{array}\right) \pi^{\prime}(y) U\left(\begin{array}{l}
\xi \\
0
\end{array}\right)=\pi^{\prime}(y) T \xi .
$$

So $T y=\pi^{\prime}(y) T$ and similarly $R y=\pi^{\prime}(y) R$, for $y \in M^{\prime}$.

Let $M$ be a von Neumann algebra with a normal faithful state $\varphi$, leading to the GNS construction $(H, \Lambda)$ (here we identify $M$ with a subalgebra of $\mathcal{B}(H)$ ). We can apply Tomita-Takesaki theory to find an anti-linear isometry $J: H \rightarrow H$ such that $M^{\prime}=J M J$ (see [20]). Let $\left(\sigma_{t}\right)_{t \in \mathbb{R}}$ be the modular automorphism group and let $\mathcal{A} \subseteq M$ be a $*$-subalgebra of elements analytic for $\left(\sigma_{t}\right)$ such that $\sigma_{z}(a) \in \mathcal{A}$ for $z \in \mathbb{C}$ and $a \in \mathcal{A}$. For $a \in \mathcal{A}$, write $a^{\prime}=J \sigma_{i / 2}(a)^{*} J$. Then

$$
a^{\prime} \Lambda(1)=J \sigma_{i / 2}(a)^{*} J \Lambda(1)=\Lambda(a) \quad(a \in \mathcal{A}) .
$$

Proposition 4.1. Let $M$ be a von Neumann algebra as above, and suppose that $\mathcal{A}^{\prime \prime}=M$. Let $N$ be a von Neumann algebra. If $\theta: N \rightarrow M$ is a completely bounded normal map, then we can find a Hilbert space $K$, normal *-homomorphisms $\pi: N \rightarrow \mathcal{B}(K)$ and $\pi^{\prime}: M^{\prime} \rightarrow \pi(N)^{\prime}$, and $\xi_{0}, \xi_{1} \in K$ such that the maps

$$
\Lambda(a) \mapsto \pi^{\prime}\left(a^{\prime}\right) \xi_{0}, \quad \Lambda(a) \mapsto \pi^{\prime}\left(a^{\prime}\right) \xi_{1} \quad(a \in \mathcal{A})
$$

are bounded and

$$
\varphi(\theta(x) a)=\left(\pi(x) \pi^{\prime}\left(a^{\prime}\right) \xi_{0} \mid \xi_{1}\right) \quad(x \in N, a \in \mathcal{A}) .
$$

Conversely, given such $K, \pi, \pi^{\prime}, \xi_{0}$ and $\xi_{1}$, there exists a completely bounded normal map $\theta: N \rightarrow M$ satisfying (4.1).

Furthermore, $\theta$ is completely positive if and only if we can choose $\xi_{0}=\xi_{1}$. 
Proof. As $\mathcal{A}^{\prime \prime}=M$, it follows that $\mathcal{A}$ is strongly dense in $M$ and hence that $\Lambda(\mathcal{A})$ is norm dense in $H$. If $\theta$ is of the form claimed, then the map $T: \Lambda(\mathcal{A}) \rightarrow K ; \Lambda(a) \mapsto$ $\pi^{\prime}\left(a^{\prime}\right) \xi_{0}$ is bounded and so extends to a bounded linear map $T: H \rightarrow K$. Similarly, there exists $R \in \mathcal{B}(H, K)$ with $R \Lambda(a)=\pi^{\prime}\left(a^{\prime}\right) \xi_{1}$. Then, for $a, b \in \mathcal{A}$ and $x \in N$,

$$
\begin{aligned}
\left(R^{*} \pi(x) T \Lambda(a) \mid \Lambda(b)\right) & =\left(\pi(x) \pi^{\prime}\left(a^{\prime}\right) \xi_{0} \mid \pi^{\prime}\left(b^{\prime}\right) \xi_{1}\right)=\left(\pi(x) \pi^{\prime}\left(\left(b^{\prime}\right)^{*} a^{\prime}\right) \xi_{0} \mid \xi_{1}\right) \\
& =\varphi\left(\theta(x) a \sigma_{-i}\left(b^{*}\right)\right),
\end{aligned}
$$

as $\left(b^{\prime}\right)^{*}=\left(J \sigma_{i / 2}(b)^{*} J\right)^{*}=J \sigma_{i / 2}(b) J=J \sigma_{i / 2}(c)^{*} J=c^{\prime}$ if $c=\sigma_{-i}\left(b^{*}\right)$, and $d \mapsto d^{\prime}$ is an anti-homomorphism. By the KMS condition, we see that

$$
\left(R^{*} \pi(x) T \Lambda(a) \mid \Lambda(b)\right)=\varphi\left(b^{*} \theta(x) a\right)=(\theta(x) \Lambda(a) \mid \Lambda(b)) .
$$

Hence $\theta$ is completely bounded, as $\theta(x)=R^{*} \pi(x) T$ for $x \in N$. If $\xi_{0}=\xi_{1}$, then $R=T$ and $\theta$ is completely positive.

Conversely, given $\theta$, from the discussion above, we can find normal $*$-homomorphisms $\pi: N \rightarrow \mathcal{B}(K)$ and $\pi^{\prime}: M^{\prime} \rightarrow \pi(N)^{\prime}$, and bounded maps $R, T: H \rightarrow K$ with $\theta(x)=R^{*} \pi(x) T$ for $x \in N$ and $R y=\pi^{\prime}(y) R, T y=\pi^{\prime}(y) T$ for $y \in M^{\prime}$. Thus, for $x \in N$ and $a \in \mathcal{A}$,

$$
\varphi(\theta(x) a)=\left(R^{*} \pi(x) T \Lambda(a) \mid \Lambda(1)\right)=\left(R^{*} \pi(x) \pi^{\prime}\left(a^{\prime}\right) T \Lambda(1) \mid \Lambda(1)\right),
$$

so the proof is complete by setting $\xi_{0}=T \Lambda(1)$ and $\xi_{1}=R \Lambda(1)$. If $\theta$ is completely positive, then we can set $R=T$, and hence $\xi_{0}=\xi_{1}$.

Notice that by the KMS condition, the calculations above also show that if $x, y \in M$ are such that $\varphi(x a)=\varphi(y a)$ for all $a \in \mathcal{A}$, then $x=y$.

The following is proved using different methods in [15] and [1]. Our proof makes explicit how $\varphi$ being tracial, for a Kac algebra, is central to the proof and indicates that understanding the modular properties of $\varphi$ for a general compact quantum group will be important in finding a completely bounded analogue of the following.

Theorem 4.2. Let $(M, \Delta)$ be a compact Kac algebra. Then there exists a splitting morphism $\theta_{*}: M_{*} \rightarrow M_{*} \widehat{\otimes} M_{*}$ such that $\theta=\theta_{*}^{*}$ is completely positive.

Proof. We have that $\varphi$ is tracial. Let $\pi: M \bar{\otimes} M \rightarrow M \bar{\otimes} M \subseteq \mathcal{B}(H \otimes H)$ be the trivial representation, let $\xi_{0}=\xi_{1}=\Lambda(1) \otimes \Lambda(1)$, and define $\pi^{\prime}$ by

$$
\pi^{\prime}(y)=(J \otimes J) \Delta(J y J)(J \otimes J) \quad\left(y \in M^{\prime}\right) .
$$

This formula is simply the natural coproduct on $M^{\prime}$; see [12, Section 4]. Let $\mathcal{A}$ be the Hopf $*$-algebra associated to $(M, \Delta)$, as before. Then we can apply the above proposition to see that there exists a completely positive normal map $\theta: M \bar{\otimes} M \rightarrow M$ such that

$$
\varphi(\theta(x) a)=\left(x(J \otimes J) \Delta\left(a^{*}\right)(J \otimes J) \Lambda(1) \otimes \Lambda(1) \mid \Lambda(1) \otimes \Lambda(1)\right),
$$

where we use that $\sigma$ is trivial, as $\varphi$ is tracial. Then $J \Lambda(a)=\Lambda(a)^{*}$ for $a \in \mathcal{A}$, and so, as $\Delta\left(a^{*}\right) \in \mathcal{A} \otimes \mathcal{A}$,

$$
\begin{aligned}
\varphi(\theta(x) a) & =\left(x(J \otimes J) \Delta\left(a^{*}\right) \Lambda(1) \otimes \Lambda(1) \mid \Lambda(1) \otimes \Lambda(1)\right) \\
& =(x(\Lambda \otimes \Lambda) \Delta(a) \mid \Lambda(1) \otimes \Lambda(1))=(\varphi \otimes \varphi)(x \Delta(a)) .
\end{aligned}
$$

In particular,

$$
\varphi(\theta \Delta(x) a)=(\varphi \otimes \varphi)(\Delta(x a))=\varphi(x a)
$$


so by the observation above, $\theta \Delta=\mathrm{id}$. Indeed, one may calculate (thinking about Proposition 3.2) that

$$
\theta\left(v_{i j}^{\alpha} \otimes v_{k l}^{\alpha}\right)=\frac{1}{n_{\alpha}} \delta_{j k} v_{i l}^{\alpha},
$$

using that $\lambda_{i}^{\alpha}=1$ for all $\alpha$ and $i$. Thus also $\Delta \theta=(\theta \otimes \mathrm{id})(\mathrm{id} \otimes \Delta)=(\mathrm{id} \otimes \theta)(\Delta \otimes \mathrm{id})$, and so $\theta_{*}$, the preadjoint to $\theta$, is a splitting morphism, as required.

If $\varphi$ is not tracial, then the above proof fails, as for $a \in \mathcal{A}$,

$$
\Delta\left(\sigma_{i / 2}(a)^{*}\right)=\left(\left(\tau_{i / 2} \otimes \sigma_{i / 2}\right) \Delta(a)\right)^{*},
$$

and hence, as $J \Lambda(b)=\Lambda\left(\sigma_{i / 2}(b)^{*}\right)$ for $b \in \mathcal{A}$,

$$
(J \otimes J) \Delta\left(\sigma_{i / 2}(a)^{*}\right)(J \otimes J)(\Lambda(1) \otimes \Lambda(1))=(\Lambda \otimes \Lambda)\left(\left(\tau_{i / 2} \sigma_{-i / 2} \otimes \mathrm{id}\right) \Delta(a)\right) .
$$

If we continue to form $\theta$ as above, then we find that

$$
\theta\left(v_{i j}^{\alpha} \otimes v_{k l}^{\beta}\right)=\delta_{\alpha \beta} v_{i l}^{\alpha} \frac{\delta_{j k}}{\operatorname{Tr}_{\alpha}} \quad\left(\alpha, \beta \in \mathbb{A}, 1 \leq i, j \leq n_{\alpha}, 1 \leq k, l \leq n_{\beta}\right) .
$$

This is nearly of the correct form, but we find that

$$
\theta \Delta\left(v_{i j}^{\alpha}\right)=\frac{n_{\alpha}}{\operatorname{Tr}_{\alpha}} v_{i j}^{\alpha} \quad\left(\alpha \in \mathbb{A}, 1 \leq i, j \leq n_{\alpha}\right) .
$$

Notice that as $n_{\alpha}=\sum_{i}\left(\lambda_{i}^{\alpha}\right)^{1 / 2}\left(\lambda_{i}^{\alpha}\right)^{-1 / 2} \leq\left(\sum_{i} \lambda^{\alpha}\right)^{1 / 2}\left(\sum_{i}\left(\lambda^{\alpha}\right)^{-1}\right)^{1 / 2}=\operatorname{Tr}_{\alpha}$, it follows that $\theta \Delta=$ id if and only if $\lambda_{i}^{\alpha}=1$ for all $\alpha, i$; that is, again, $\varphi$ is tracial. It is curious that exactly the same formula, in a seemingly rather different context, arises in [4, Theorem 4.5]. The remarks in [4] after this theorem are also of interest and suggest that a simple "rescaling" argument is unlikely to be able to turn $\theta$ into a map with the required properties.

\section{REFERENCES}

[1] O. Yu. Aristov, Amenability and compact type for Hopf-von Neumann algebras from the homological point of view. In Banach algebras and their applications, Contemp. Math., 363, 15-37. Amer. Math. Soc., Providence, RI, 2004. MR2097947(2006f:46055)

[2] O. Yu. Aristov, Biprojective algebras and operator spaces, J. Math. Sci. (New York) 111 (2002), 3339-3386. MR.1847551 (2003f:46090)

[3] E. Bédos, G. J. Murphy, and L. Tuset, Co-amenability of compact quantum groups, J. Geom. Phys. 40 (2001), 130-153. MR1862084 (2002m:46100)

[4] E. Bédos, G. J. Murphy, and L. Tuset, Amenability and co-amenability of algebraic quantum groups. II, J. Funct. Anal. 201 (2003), 303-340. MR.1986692 (2004e:46085)

[5] E. Christensen and A. M. Sinclair, Module mappings into von Neumann algebras and injectivity, Proc. London Math. Soc. (3) 71 (1995), 618-640. MR.1347407 (96m:46107)

[6] E. G. Effros and Z.-J. Ruan, Operator spaces, London Math. Society Monographs, New Series, 23. The Clarendon Press, Oxford University Press, New York, 2000. MR 1793753 (2002a:46082)

[7] M. Enock and J.-M. Schwartz, Kac algebras and duality of locally compact groups, SpringerVerlag, Berlin, 1992. MR1215933 (94e:46001)

[8] U. Haagerup and M. Musat, Classification of hyperfinite factors up to completely bounded isomorphism of their preduals, J. Reine Angew. Math. 630 (2009), 141-176. MR.2526788

[9] A. Ya. Helemskii, The homology of Banach and topological algebras, translated from the Russian by Alan West. Mathematics and Its Applications (Soviet Series), 41. Kluwer Academic Publishers Group, Dordrecht, 1989. MR.1093462 (92d:46178)

[10] J. Kustermans, Locally compact quantum groups. In Quantum independent increment processes. I, Lecture Notes in Math., 1865. Springer, Berlin, 2005, 99-180. MR2132094 (2006f:46073) 
[11] J. Kustermans and S. Vaes, Locally compact quantum groups, Ann. Sci. École Norm. Sup. (4) 33 (2000), 837-934. MR1832993 (2002f:46108)

[12] J. Kustermans and S. Vaes, Locally compact quantum groups in the von Neumann algebraic setting, Math. Scand. 92 (2003), 68-92. MR.1951446 (2003k:46081)

[13] A. Maes and A. Van Daele, Notes on compact quantum groups, Nieuw Arch. Wisk. (4) 16 (1998), 73-112. MR1645264(99g:46105)

[14] V. I. Paulsen, Completely bounded maps and dilations, Pitman Research Notes in Mathematics Series, 146. Longman Scientific \& Technical, Harlow; John Wiley \& Sons, Inc., New York, 1986. MR868472 (88h:46111)

[15] Z.-J. Ruan and G. Xu, Splitting properties of operator bimodules and operator amenability of Kac algebras. In Operator theory, operator algebras and related topics (Timişoara, 1996), 193-216, Theta Found., Bucharest, 1997. MR.1728421 (2000j:46113)

[16] V. Runde, Biflatness and biprojectivity of the Fourier algebra, Arch. Math. (Basel) 92 (2009), 525-530. MR2506954

[17] V. Runde, Lectures on amenability, Lecture Notes in Math., 1774. Springer-Verlag, Berlin, 2002. MR1874893 (2003h:46001)

[18] P. M. Sołtan, Quantum Bohr compactification, Illinois J. Math. 49 (2005), 1245-1270. MR2210362 (2007a:46079)

[19] M. Takesaki, Theory of operator algebras. I, reprint of the first (1979) edition. Encyclopaedia of Mathematical Sciences, 124. Operator Algebras and Non-commutative Geometry, 5. Springer-Verlag, Berlin, 2002. MR1873025 (2002m:46083)

[20] M. Takesaki, Theory of operator algebras. II, Encyclopaedia of Mathematical Sciences, 125. Operator Algebras and Non-commutative Geometry, 6. Springer-Verlag, Berlin, 2003. MR1943006 (2004g:46079)

[21] T. Timmermann, An invitation to quantum groups and duality. From Hopf algebras to multiplicative unitaries and beyond. European Mathematical Society (EMS), Zürich, 2008. MR2397671 (2009f:46079)

[22] J. Tomiyama, On the projection of norm one in $W^{*}$-algebras, Proc. Japan Acad. 33 (1957), 608-612. MR0096140 (20:2635)

[23] P. J. Wood, The operator biprojectivity of the Fourier algebra, Canad. J. Math. 54 (2002), 1100-1120. MR1924715 (2003j:46085)

[24] S. L. Woronowicz, Compact quantum groups. In Symétries quantiques (Les Houches, 1995), 845-884. North-Holland, Amsterdam, 1998. MR.1616348 (99m:46164)

[25] S. L. Woronowicz, Compact matrix pseudogroups, Comm. Math. Phys. 111 (1987), 613-665. MR.901157 (88m:46079)

[26] S. L. Woronowicz, Twisted SU(2) group. An example of a noncommutative differential calculus, Publ. Res. Inst. Math. Sci. 23 (1987), 117-181. MR890482 (88h:46130)

School of Mathematics, University of Leeds, Leeds, LS2 9JT, United Kingdom

E-mail address: matt.daws@cantab.net 